\title{
The Predictive Value of Procalcitonin in the Prognosis of Patients with Acute Coronary Syndrome
}

\author{
(1) Abuzer Coșkun¹, (1) Can Aktaș², (1) Șevki Hakan Eren³
}

${ }^{1}$ Clinic of Emergency Service, Sivas State Hospital, Sivas, Turkey

2Department of Emergency Medicine, Koç University Faculty of Medicine, İstanbul, Turkey

${ }^{3}$ Department of Emergency Medicine, Gaziantep University Faculty of Medicine, Gaziantep, Turkey

\begin{abstract}
Aim: The study aimed to investigate the prognostic value of serum procalcitonin (PCT) levels in patients with acute coronary syndrome (ACS).

Materials and Methods: Two hundred patients with ACS who applied to the emergency department due to chest pain and were hospitalized into the cardiology clinic of our hospital between January 2014 and December 2016 were included in this cross-sectional cohort study. The patients were divided into four groups based on their diagnosis. The first group was inferior myocardial infarction (MI), the second group was anterior MI, the third group was non-ST-elevation MI (NSTEMI), and the fourth group was high-risk unstable angina (UA). These groups were compared according to PCT, age, gender, left ventricular ejection fraction, cardiac troponin (cTn) I, three-vessel disease (TVD), and mortality rate.

Results: There were statistically significant differences between ACS groups concerning PCT values. PCT level was significantly higher in inferior MI than in anterior MI, NSTEMI, and UA groups. There were statistically significant differences between cTn positivity checked at $0^{\text {th }}, 6^{\text {th }}$, and $12^{\text {th }}$ hours and ischemic heart failure after MI as well as between mortality and TVD. TVD and mortality were found to be significantly higher in the inferior MI group than the other subgroups. Ischemic heart failure was found to be statistically higher in anterior subgroup than the other subgroups.
\end{abstract}

Conclusion: Increased PCT level may be a marker that can be used in indicating ACS and its prognosis.

Keywords: Acute Coronary syndrome, emergency department, mortality, procalcitonin

\section{Introduction}

Acute coronary syndrome (ACS) including unstable angina (UA), non-ST-elevation myocardial infarction (NSTEMI) and ST-elevation myocardial infarction (STEMI) continue to be the main reason of mortality and morbidity all around the world despite the advancing medical technologies, many factors revealed in the ethiopathogenesis and developments in coronary artery disease (CAD) treatment. Many studies on atherosclerotic disease that has increasing prevalence are conducted and new results are obtained (1). Cardiac troponin (CTn) is quite sensitive and is a specific indicator of myocardial damage. Increased levels of cTn have importance in ACS concerning prognosis and course of the treatment. Therefore, measurements of cTn levels are often used for differential diagnosis in ACS in the emergency department (ED) and intensive care units (2-5). However, it is impossible to detect the patients with ACS who have high risk via serum troponins, because myocardial necrosis is not seen in many of these patients. Therefore, new cardiac biomarkers, which will help rapid and absolute diagnosis, are required in order for risk evaluation in the patients with ACS, before the traditional markers, which indicate myocardial cell damage and in case where these markers do not 
elevate. It is a known fact that inflammation plays a vital role in every step of atherosclerosis in the point where there are theories that have been developed and studies that have been conducted on the pathogenesis of atherosclerosis for over a hundred years. Inflammation theory is mostly accepted today (1,6-9). There are several studies for cardiac markers except for cTn today, and one of them is procalcitonin (PCT). PCT is the pre-hormone of calcitonin, which is involved in calcium homeostasis and which is normally secreted by the $C$ cells of the thyroid glands with response to hypercalcemia under physiologic conditions (10). PCT is known to be a diagnostic marker for severe sepsis or septic shock in critically ill patients (11). In sepsis, PCT is thought to be produced by the liver and peripheral blood mononuclear cells modulated by lipopolysaccharides and sepsis-related cytokines (12). The PCT level also increases in other clinical settings such as trauma, major cardiac surgery, pancreatitis, and cardiogenic shock. There are still contrasting data about the role of PCT in patients with acute myocardial infarction in those with ACSS, which are NSTEMI or UA (13). The relationship between ACS and PCT was searched in certain studies before, but we could not find any study that searched the relationship between subgroups of ACS and PCT in the literature. We aimed to evaluate inferior, anterior myocardial infarction (MIS), which are subgroups of STEMI, NSTEMI as well as UA and PCT levels, the relationship between them and CTn, troponin level, ischemic heart failure (IHF), which is one of the most frequent complications after acute MI (AMI) with regard to three-vessel disease (TVD) and mortality, and to indicate whether PCT is a predictive marker for these cases or not in our study.

\section{Materials and Methods}

\section{Study Design and Population}

The study was prospectively conducted throughout six months. Two hundred patients with ACS applied to the ED of Sivas Numune Hospital between January 2014 and December 2016. The patients were divided into four groups, each of which consisted of 50 individuals. The first group included inferior AMI, inferolateral AMI, inferoposterior AMI and right ventricle MI; the second group included septal AMI, anterior AMI, lateral AMI, high lateral AMI and common anterior AMI; the third group included NSTEMI, and the fourth group included the patients with UA who have high risk based on the Braunwald classification (14). Patient-focused medical history, physical examination, twelvelead electrocardiography (ECG), and chest radiography were conducted and the results were recorded. Venous blood samples were obtained from the patients to measure cardiac biomarkers and PCT levels. TVD was considered when left anterior descending artery, circumflex artery, and right coronary arteries had as at least $50 \%$ occlusion. The patients who had chest pain and/or discomfort lasting at least 30 minutes and ECG with STEMI following the American College of Cardiology Foundation (ACCF)/ American Heart Association (AHA) 2013 guideline were included in the study (15). UA/NSTEMI was defined according to the criteria of the AHA/ACCF 2014 guideline for the management of patients with non-ST-elevation ACS. All of the patients were checked through transthoracic echocardiography (TTE) to determine whether there were focal wall motion abnormalities or not. Philips Epiq 7 ultrasound machine was used for TTE in this study.

All patients gave written informed consent, and the study was approved by the Ethical Committee of Faculty of Medicine of Cumhuriyet University (date: 17/01/2018, decision no: 201801726).

\section{Procalcitonin}

PCT was examined via a compact automated immunoassay system (Mini Vidas', BioMérieux, Marcy-l'Étoile, France) within 20-30 minutes. The results within 0-0.05 ng/mL were accepted as normal.

\section{Cardiac Biomarker Analysis}

Venous blood samples were obtained from the antecubital veins of the patients in order to measure serum levels of troponin I. Elecsys troponin I STAT (cobas e 411 analyzer, Roche Diagnostics $\mathrm{GmbH}$, Mannheim, Germany) was used to measure troponin I levels. CTnl levels of patients were measured at $0^{\text {th }}, 6^{\text {th }}$, and $12^{\text {th }}$ hours.

\section{Angiographic Analysis}

Percutaneous coronary intervention studies of all patients were evaluated by cardiologists who were blind to patients' clinical and cardiac marker status.

\section{Statistical Analysis}

The data obtained from this study were analysed via SPSS 20 software package. Shapiro-Wilk test was used as the test of normality. Mann-Whitney $\mathrm{U}$ and Kruskal-Wallis $\mathrm{H}$ tests were used to compare the differences between groups since the variables were non-normally distributed. The group that caused the significance was determined using multiple-comparison posthoc test. Friedman's two-way ANOVA was used in the analysis of more than two non-normally distributed variables and multiplecomparison post-hoc test was used to detect variables that varied from each other. Chi-square analysis was used for categorical variables. Fisher's exact test was used in cases where estimated values in sources in $2 \times 2$ tables had not sufficient volume. Pearson's chi-square analysis was applied through Monte Carlo Simulation in RxC tables. The significance level was used as 0.05 . $\mathrm{P}<0.05$ was considered as statistically significant. 


\section{Results}

The clinical and demographic characteristics of the patients are listed in Table 1. cTn values of inferior, anterior, and NSTEMI groups at the $0^{\text {th }}, 6^{\text {th }}$, and $12^{\text {th }}$ hours were found significantly higher than cTnl value of UA group. cTn value of the anterior group at the $12^{\text {th }}$ hour was found significantly higher than the cTnI value of the NSTEMI group (Table 2). There was no statistically significant difference between ACS groups concerning PCT values.

PCT level was found statistically higher in the inferior MI group

Table 1. Baseline characteristics of study patients and value distributions of variables

\begin{tabular}{|c|c|c|c|c|c|c|c|}
\hline \multicolumn{8}{|c|}{ Acute coronary } \\
\hline & \multirow[t]{2}{*}{ All patients } & \multicolumn{3}{|c|}{ Patients with } & \multirow[b]{2}{*}{ UA } & \multirow[b]{2}{*}{$\mathrm{H}$} & \multirow[b]{2}{*}{ p-value } \\
\hline & & IMI & AMI & NSTEMI & & & \\
\hline \multicolumn{8}{|c|}{ Basaline characteristcis } \\
\hline Age, mean $\pm S D, y r$ & $63.5 \pm 9.4$ & $64.4 \pm 8.3$ & $63.9 \pm 10.5$ & $63.7 \pm 9.2$ & $62.1 \pm 9.7$ & 1.53 & 0.675 \\
\hline Sex, Female/male & $74 / 126$ & $20 / 30$ & $21 / 29$ & $19 / 31$ & $14 / 36$ & $x^{2}=2.488$ & 0.477 \\
\hline \multicolumn{8}{|l|}{ Labaratory finding } \\
\hline BS, mg/dL & $164 \pm 87$ & $195 \pm 114$ & $164 \pm 80$ & $155 \pm 61$ & $143 \pm 80$ & 9.810 & 0.020 \\
\hline PCT, ng/mL & $0.4 \pm 0.6$ & $0.5 \pm 0.6$ & $0.6 \pm 0.7$ & $0.3 \pm 0.5$ & $0.06 \pm 0.06$ & 135.2 & 0.001 \\
\hline LVEF, \% & $48.8 \pm 12$ & $46.7 \pm 11.8$ & $3.3 \pm 11.6$ & $48.8 \pm 11.3$ & $56.6 \pm 9.4$ & 34.8 & 0.001 \\
\hline $\mathrm{TG}, \mathrm{mg} / \mathrm{dL}$ & $156 \pm 90.4$ & $165.8 \pm 65.7$ & $142.7 \pm 85.2$ & $153.6 \pm 96.4$ & $162 \pm 9.4$ & 5.801 & 0.121 \\
\hline $\mathrm{CHOL}, \mathrm{mg} / \mathrm{dL}$ & $198.4 \pm 85.7$ & $206.6 \pm 69$ & $225.8 \pm 112.2$ & $188.1 \pm 91.2$ & $172.9 \pm 49.8$ & 9.932 & 0.019 \\
\hline HDL, mg/dL & $33.6 \pm 8,8$ & $32.3 \pm 5.8$ & $33.6 \pm 7.2$ & $35.3 \pm 12.6$ & $33.3 \pm 7.9$ & 1.756 & 0.625 \\
\hline LDL, mg/dL & $116,6 \pm 4.9$ & $124,5 \pm 40$ & $130.4 \pm 66.2$ & $105.5 \pm 43.1$ & $106.2 \pm 37.8$ & 7.619 & 0.055 \\
\hline cTn 1, ng/mL & $2.5 \pm 4$ & $3.5 \pm 2.3$ & $4.4 \pm 6.6$ & $2.2 \pm 2.2$ & $0.05 \pm 0.1$ & 113.6 & 0.001 \\
\hline cTn 2 & $6.8 \pm 7.3$ & $9.4 \pm 5.2$ & $10.8 \pm 8.4$ & $6.8 \pm 7$ & $0.1 \pm 0.2$ & 119.4 & 0.001 \\
\hline cTn 3 & $16.6 \pm 17.8$ & $23.4 \pm 13.4$ & $27.5 \pm 21.4$ & $15.3 \pm 14.5$ & $0.3 \pm 0.5$ & 121.1 & 0.001 \\
\hline AST, IU/L & $32 \pm 29$ & $34.8 \pm 38$ & $33.3 \pm 39.5$ & $28.6 \pm 13.9$ & $31.3 \pm 13.6$ & 6.270 & 0.099 \\
\hline ALT, IU/L & $32.3 \pm 34.5$ & $33.3 \pm 41.7$ & $35 \pm 46.3$ & $14.6 \pm 16.2$ & $36.4 \pm 24.6$ & 9.397 & 0.024 \\
\hline ALP, IU/L & $109.5 \pm 55.4$ & $125 \pm 48$ & $100.6 \pm 43.7$ & $115.4 \pm 56.3$ & $97.1 \pm 67.6$ & 17.401 & 0.001 \\
\hline CK, IU/L & $160.8 \pm 109.4$ & $149.4 \pm 67.2$ & $158.3 \pm 160.2$ & $173.1 \pm 98.1$ & $162.6 \pm 92.2$ & 4.325 & 0.228 \\
\hline CKMB, IU/L & $32.5 \pm 20.8$ & $33.9 \pm 20.2$ & $30.4 \pm 25.4$ & $34 \pm 17.9$ & $31.6 \pm 19.3$ & 3.693 & 0.297 \\
\hline WBC, $10^{\wedge} 3 / \mathrm{UL}$ & $10.8 \pm 3.5$ & $9.89 \pm 3.25$ & $10.2 \pm 3.7$ & $11.3 \pm 3.1$ & $11 \pm 3.8$ & 3.357 & 0.340 \\
\hline hsCRP, mg/dL & $5.3 \pm 5.3$ & $7.4 \pm 5.8$ & $5.7 \pm 5$ & $4.6 \pm 5.8$ & $3.5 \pm 3.9$ & 26.080 & 0.001 \\
\hline \multicolumn{8}{|c|}{$\begin{array}{l}\text { IMI: Inferior myocardial infarction, AMI: Anterior myocardial infarction, NSTEMI: Non-ST elevation myocardial infarction, UA: Unstable angina, BS: Blood suger, PCT: Procalcitonin, } \\
\text { LVEF: Left ventricular ejection fraction, TG: Triglycerides, CHOL: Cholesterol, HDL: High density lipoprotein, LDL: Low density lipoprotein, SD: Standard deviation, cTn: Troponin, } \\
\text { AST: Aspartate aminotransferase, ALT: Alanine aminotransferase, ALP: Alkaline phosphatase, CK: Creatine kinase, CKMB: Creatine kinase-MB, WBC; White blood cell, hsCRP: } \\
\text { High-sensitivity C-reactive protein, yr: year, } p<0.05\end{array}$} \\
\hline
\end{tabular}

Table 2. Chi-square analysis of varieties of Acute Coronary syndrome according to variables

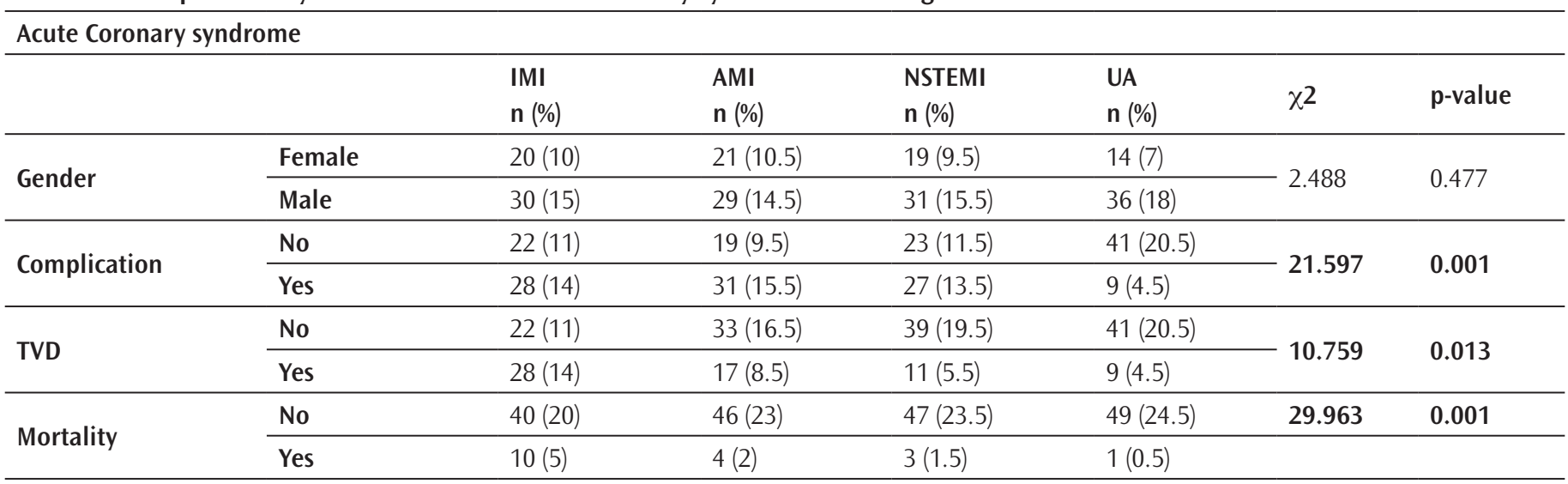


than MI, NSTEMI, and UA groups. Left ventricular ejection fraction (LVEF) levels were found statistically lower in the inferior $\mathrm{MI}$, anterior MI, and NSTEMI groups than the UA group. There was no statistically significant difference between cTnI positivity, which as checked at the $0^{\text {th }}, 6^{\text {th }}$, and $12^{\text {th }}$ hours and IHF, which occurred after AMI, as well as between mortality and TVD (Table 3). There was no statistically significant difference based on PCT and concerning progressing IHF, TVD, and mortality (Table 3). In terms of PCT, ACS, TVD, IHF, mortality, and cTn levels were found as a prognostic symptom in univariate analysis. Gender, age, and blood glucose levels were statistically insignificant. However, in multivariate linear regression analysis with the advanced staged method, ACS, TVD, and IHF were associated with increased CAD risk after adjustment for statistically significant variables in univariate analysis (Table 4). In terms of mortality, ACS, TVD, IHF, $\mathrm{PCT}$, and gender were found as prognostic factors in univariate analysis. Age, cTn, and blood glucose levels were statistically insignificant. However, in multivariate linear regression analysis with the advanced staged method, ACS, TVD, PTC, and IHF were associated with increased CAD risk after adjustment for statistically significant variables in univariate analysis (Table 5).

Table 3. Analysis of the variables that develop after acute coronary syndrome according to troponin and procalcitonin levels

\begin{tabular}{|c|c|c|c|c|c|c|c|c|c|c|}
\hline & & & \multicolumn{4}{|c|}{ Troponin levels } & \multicolumn{4}{|c|}{ Proclacitonin levels } \\
\hline & & & $\mathrm{n}$ & SD & $Z$ & $p$-value & & SD & $\mathrm{Z}$ & p-value \\
\hline \multirow{6}{*}{ 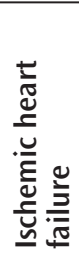 } & \multirow{2}{*}{ 0. h cTn } & No & 105 & $1.4 \pm 1.9$ & \multirow{2}{*}{-5.889} & \multirow{2}{*}{0.001} & & \multirow{3}{*}{$0.28 \pm 0.52$} & \multirow{6}{*}{-2.97} & \multirow{6}{*}{0.003} \\
\hline & & Yes & 95 & $3.8 \pm 5.2$ & & & & & & \\
\hline & \multirow{2}{*}{ 6. h cTn } & No & 105 & $4.4 \pm 5.7$ & \multirow{2}{*}{-5.547} & \multirow{2}{*}{0.001} & No & & & \\
\hline & & Yes & 95 & $9.4 \pm 8.0$ & & & & & & \\
\hline & \multirow{2}{*}{ 12. h cTn } & No & 105 & $10.7 \pm 13.1$ & \multirow{2}{*}{--5.654} & \multirow{2}{*}{0.001} & \multirow{2}{*}{ Yes } & \multirow{2}{*}{$0.45 \pm 0.61$} & & \\
\hline & & Yes & 95 & $23.2 \pm 19.9$ & & & & & & \\
\hline \multirow{6}{*}{$\begin{array}{l}\frac{Z}{\frac{Z}{\pi}} \\
\frac{ \pm}{ \pm} \\
\sum\end{array}$} & \multirow{2}{*}{ 0. h cTn } & No & 182 & $2.0 \pm 2.3$ & \multirow{2}{*}{-4.514} & \multirow{2}{*}{0.001} & \multirow{3}{*}{ - No } & \multirow{3}{*}{$0.31 \pm 0.53$} & \multirow{6}{*}{--5.64} & \multirow{6}{*}{0.001} \\
\hline & & Yes & 18 & $7.7 \pm 9.9$ & & & & & & \\
\hline & \multirow{2}{*}{ 6. h cTn } & No & 182 & $5.8 \pm 5.6$ & \multirow{2}{*}{-4.212} & \multirow{2}{*}{0.001} & & & & \\
\hline & & Yes & 18 & $16.7 \pm 12.8$ & & & & & & \\
\hline & \multirow{2}{*}{ 12. h cTn } & No & 182 & $14 \pm 13.8$ & \multirow{2}{*}{-4.553} & \multirow{2}{*}{0.001} & \multirow{2}{*}{ Yes } & \multirow{2}{*}{$0.93 \pm 0.69$} & & \\
\hline & & Yes & 18 & $43.5 \pm 28.8$ & & & & & & \\
\hline \multirow{6}{*}{ 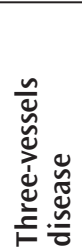 } & $0 \mathrm{hcTn}$ & No & 135 & $2.1 \pm 4.4$ & -4381 & 0001 & & & & \\
\hline & 0. n cin & Yes & 65 & $3.4 \pm 2.8$ & -4.381 & 0.001 & No & $021+047$ & & \\
\hline & $6 \mathrm{~h} \subset \mathrm{Tn}$ & No & 135 & $5.4 \pm 6.8$ & 4587 & 0001 & No & $0.24 \pm 0.4 /$ & -425 & 0001 \\
\hline & $0.11 \mathrm{c}$ & Yes & 35 & $9.7 \pm 7.4$ & -4.007 & 0.001 & & & -4.25 & 0.001 \\
\hline & $12 \mathrm{~h} r \mathrm{Tn}$ & No & 135 & $13.8 \pm 17.9$ & 281 & 0001 & Yes & $061+068$ & & \\
\hline & $12.110 \mathrm{CII}$ & Yes & 35 & $22.4 \pm 17.8$ & -4.281 & 0.001 & res & $0.61 \pm 0.68$ & & \\
\hline
\end{tabular}

SD: Standard deviation

Table 4. Univariate and multivariate linear regression analyses for predicting the development of Procalcitonin

\begin{tabular}{|c|c|c|c|c|c|c|c|c|c|c|c|c|}
\hline \multicolumn{13}{|c|}{ Procalcitonin } \\
\hline & \multicolumn{5}{|c|}{ Univariate } & \multicolumn{5}{|c|}{ Multivariate } & \multicolumn{2}{|c|}{ Correlation } \\
\hline & RS & $\mathbf{F}$ & $\beta$ & $\mathrm{t}$ & $p$-value & RS & $\mathrm{F}$ & $\beta$ & $t$ & $p$-value & $r$ & p-value \\
\hline ACS & 0.43 & 146.95 & 1.2 & 15.87 & 0.001 & \multirow{6}{*}{0.63} & \multirow{6}{*}{26.38} & -0.30 & -10.70 & 0.001 & -0.653 & 0.001 \\
\hline TVD & 0.11 & 25.09 & 0.23 & 4.95 & 0.001 & & & 0.06 & 2.62 & 0.010 & 0.335 & 0.001 \\
\hline cTnI & 0.06 & 11.70 & 0.28 & 5.88 & 5.88 & & & & & & 0.236 & 0.001 \\
\hline cTnII & 0.11 & 23.72 & 0.19 & 3.58 & 0.001 & & & & & & 0.327 & 0.001 \\
\hline cTnIII & 0.11 & 25.30 & 0.18 & 3.46 & 0.001 & & & & & & 0.337 & 0.001 \\
\hline Age & 0.00 & 0.2 & 0.24 & 0.86 & 0.388 & & & & & & & \\
\hline
\end{tabular}


Table 5. Univariate and multivariate linear regression analyses for predicting the development of Mortality

\begin{tabular}{|c|c|c|c|c|c|c|c|c|c|c|}
\hline \multicolumn{11}{|l|}{ Mortality } \\
\hline & \multicolumn{5}{|c|}{ Univariate } & \multicolumn{5}{|c|}{ Multivariate } \\
\hline & RS & $F$ & $\beta$ & $t$ & p-value & RS & $F$ & $\beta$ & $\mathrm{t}$ & p-value \\
\hline ACS & 0.05 & 11.19 & -0.23 & -3.34 & 0.001 & \multirow{10}{*}{0.37} & \multirow{10}{*}{11.03} & -0.14 & -3.53 & 0.006 \\
\hline TVD & 0.05 & 10.37 & 0.22 & 3.22 & 0.001 & & & 0.11 & 3.02 & 0.037 \\
\hline Mortality & 0.30 & 86.08 & 0.55 & 9.28 & 0.001 & & & 0.53 & 8.31 & 0.001 \\
\hline IHF & 0.06 & 12.23 & 0.24 & 3.50 & 0.001 & & & 0.21 & 3.36 & 0.001 \\
\hline cTnl & 0.00 & 0.17 & 0.03 & 0.41 & 0.680 & & & & & \\
\hline cTnII & 0.01 & 2.6 & 0.12 & 1.66 & 0.098 & & & & & \\
\hline cTnIII & 0.01 & 2.40 & 0.11 & 1.55 & 0.123 & & & & & \\
\hline Age & 0.00 & 0.24 & 0.04 & 0.49 & 0.626 & & & & & \\
\hline Gender & 0.02 & 4.34 & 0.15 & 2.08 & 0.39 & & & & & \\
\hline BS & 0.01 & 2.35 & 0.11 & 1.53 & 0.127 & & & & & \\
\hline
\end{tabular}

\section{Discussion}

The data on PCT in ACS are limited and controversial today. The number of the studies on relationships between PCT levels and inferior AMI; anterior AMI, NSTEMI, and UA groups; PCT levels and IHF, TVD and mortality occurring in ACS in ED admissions were less when the literature was reviewed. Therefore, we conducted our study in order to detect these relationships.

Kafkas et al. (16) measured PCT, II-6, creatine kinase-MB (CKMB), troponin I and C-reactive protein levels in AMI at the time of admission and certain hours and found that PCT level was higher in patients with AMI. Erren et al. (17) indicated that PCT levels were associated with the level of atherosclerosis in cases with CAD and peripheral artery disease. Unlike the other studies, we detected that the PCT level was significantly high in ACS. Atherosclerosis, which is an inflammatory disease, was available based on CAD (18). Biasucci et al. (10) suggested that PCT was released and its level increased in patients with ACS based on the inflammatory processes that occur during AMI in their studies. In similar studies, it was revealed that PCT was an inflammatory marker, and its level increased in patients with AMI $(19,20)$. We detected that PCT increased at a lower level in the UA group, where inflammation was less than the other groups in our study. However, we found that PCT levels were significantly high in STEMI and NSTEMI. These results support the articles stating that PCT may increase based on the inflammatory processes that occur after ACS. The diagnosis of ACS is essential and valuable in determining its prognosis and planning its treatment. It has been found out that the increased PCT levels are associated with a poorer prognosis for CAD, and PCT levels are correlated with the level of atherosclerosis in patients with CAD in recent studies
(10,16,21-23). Sinning et al. (24) reported that the PCT levels, which increased at the beginning, are associated with the rate of the cardiovascular case and a higher level of mortality, which increased in the follow-up. Ataoglu et al. (12) measured PCT levels in patients with ACS at the time of application and after 48 hours, and stated that the high level of PCT concentration indicated the increased 6-month-mortality in patients with severe myocardial damage upon infarction in 48 hours after the application. In a study indicating the relationship between troponin and mortality, Antman et al. (25) reported that a high level of cTnl in patients with $\mathrm{Ml}$ is a good indicator in predicting the possible rate of death.

We detected a significant relationship between PCT level at the time of admission and mortality in our study. We also detected this relationship in cTn levels studied at $6^{\text {th }}$, and $12^{\text {th }}$ hours. Unlike the literature, we found that PCT and CTnl levels were associated with the increased mortality in ACS.

IHF is one of the most frequent complications of occurring after MI. Rao et al. (26) detected a strong negative correlation between LVEF and cTnT levels in their study on 50 patients with AMI, whose LVEF was under 40\%. Accordingly, LVEF may decrease, and CTnT levels may increase at the same rates based on the extent of the infarction site. In a study indicating the relationship between IHF and PCT, Remskar et al. (27) detected that the PCT level increased in complications such as pulmonary edema and cardiogenic shock. We detected IHF, UA, and NSTEMI based on STEMI at a higher level in our study. We found LVEF was at a lower rate in patients with anterior MI among the STEMI subgroups. We detected a significant relationship between heart failure of both the high level of CTnI and PCT values in the evaluation of the relationship between heart failure and both CTnI and PCT. 
Particularly the increased level of PCT in heart failure leads to consider that inflammation is an important pathophysiological factor and may affect the prognosis of the patient. In general, the prognosis of CAD is related to the number of affected veins (one-, two-or TVD) and the degree of dysfunction of the left ventricle. A patient with a single affected vein and well left ventricular function have an excellent outcome (5-year survival >90\%), whereas a patient with severe left ventricular dysfunction and extensive disease involving three veins has a poor prognosis (28). Since the number of the involved vein has an effect on prognosis, PCT, and CTnl levels of patients who applied due to ACS and in whom TVD was detected after angiography were examined in our study. It was detected that the increase in CTn and PCT levels was higher in patients with TVD and associated with mortality at the same time (21). We consider that PCT is a prognostic factor such as troponin in our study unlike the previous studies according to these data. We also divided ACS into subgroups and examined the relationship between ACS subgroups in our study unlike the other studies. As a result of this, we detected that the PCT level in inferior MI groups was significantly higher than the other subgroups. We detected that TVD and mortality levels in inferior MI groups were significantly high when we examined the relationship between IHF, TVD, and mortality levels of these subgroups. We consider that PCT is a prognostic factor, such as troponin on the contrary to the previous studies in our study, which we conducted based on these data. It should be acknowledged that there are several limitations related to the present study. For instance, this study was conducted in a single center with a small patient group, and the patients were not divided into groups based on their treatment characteristics. A multi-centric study with more patients grouping based on their treatments and with a long-term follow-up can reveal the importance of PCT.

\section{Conclusion}

We detected that both CTnl levels and PCT levels increased in CAD in this study. PCT level increased at a lower level than other groups in cases where the level of inflammation such as UA was low, and it also increased at a lower level in NSTEMI than STEMI. This case makes us consider that PCT is correlated with the level of atherosclerosis in patients with CAD, and PCT is released based on the inflammatory processes occurring during $\mathrm{MI}$. We detected that PCT and troponin levels increased in the inferior subgroup among ACS groups in cases such as TVD involvement and mortality affecting the prognosis of the patient. Moreover, IHF was found to be significantly high in the anterior subgroup. We consider that PCT is also a prognostic factor like CTnI. As a result, the increased level of PCT can be a marker that will be used in indicating both ACS and its prognosis.

\section{Ethics}

Ethics Committee Approval: The study was approved by the Ethical Committee of Faculty of Medicine of Cumhuriyet University (date: 17/01/2018, decision no: 2018-01726).

Informed Consent: All patients gave written informed consent.

Peer-review: Externally and internally peer-reviewed.

\section{Authorship Contributions}

Medical Practices: A.C., C.A., Concept: A.C., Design: A.C., S.H.E., Data Collection or Processing: A.C., Analysis or Interpretation: A.C., C.A., Literature Search: A.C., SHE., Writing: A.C., C.A.

Conflict of Interest: No conflict of interest was declared by the authors.

Financial Disclosure: The authors declared that this study received no financial support.

\section{References}

1. World Health Organisation. World healt report, making difference, Geneva WHO 1999

2. Bayes-Genis A, Conover CA, Overgaard MT, Bailey KR, Christiansen M, Holmes DR Jr, et al. Pregnancy-associated plasma protein A as a marker of acute coronary syndromes. N Engl J Med. 2001;345:1022-9.

3. Gendrel D, Bohuon C. Procalcitonin as a marker of bacterial infection Pediatr Infect Dic J. 2000;19:679-88.

4. Maisner M. Procalcitonin-a new, innovative infection parameter biochemical andclinical aspects. 3. Revised and expanded edition. (Thieme, Stuttgart, New York), 2000

5. Braunwald E, Antman EM, Beasley JW, Califf RM, Cheitlin MD, Hochman JS, et al; American College of Cardiology; American Heart Association. Committee on the Management of Patients With Unstable Angina. ACC/AHA 2002 guideline update for the management of patients with unstable angina and non-ST-segment elevation myocardial infarction--summary article: a report of the American College of Cardiology/American Heart Association task force on practice guidelines (Committee on the Management of Patients With Unstable Angina). J Am Coll Cardiol. 2002;40:1366-74.

6. Sheehan P, Vasikaran SD. The evolving clinical role of cardiac troponins and new acute miyokardial infarction guidelines: Implications for the clinical laboratory. Clin Bichemist Rev. 2001;23:52-65.

7. Wu AHB. Increased troponin in patients with sepsis and septic shock: myocardial necrosis or reversible myocardial depression? Intensive Care Med. 2001;27:959-61.

8. Morow DA, Rıfai N, Tanasijevic MJ, Wybenga DR, de Lemos JA, Antman EM Clinical efficasy of three assays for cardiac troponin I for risk stratification in acute coronary syndroms: A trombolysis in myocardial infarction (TIMI) IIB substudy. Clin Chem. 2000;46:453-60.

9. Jaffe AS. A biomarker odyssey. Clin Chim Acta. 1999;284:197-211.

10. Biasucci LM, Biasillo G, Stefanelli A. Procalcitonin and acute coronary syndromes: a new biomarker for an old disease. Intern Emerg Med. 2009;4:363-5.

11. Uzzan B, Cohen R, Nicolas P, Cucherat M, Perret GY. Procalcitonin as a diagnostic test for sepsis in critically ill adults and after surgery or trauma: a systematic review and meta-analysis. Crit Care Med. 2006;34:1996-2003.

12. Ataoglu HE, Yilmaz F, Uzunhasan I, Cetin F, Temiz L, Döventaș YE, et al Procalcitonin: a novel prognostic marker in cardiac disease. J Int Med Res. 2010;38:52-61. 
13. Ertem AG, Efe TH, Yayla C, Akboğa MK, Açar B, Ünal S, et al. The Association Between Serum Procalcitonin Levels and Severity of Coronary Artery Disease Assessed by SYNTAX Score in Patients With Acute Coronary Syndrome. Angiology. 2017;68:40-5.

14. Cannon CP, Braunwald E. Unstable angina. In Braunwald Heart Disease 6 th ed. Philadelphia W. B. Saunders Company. 2001:1232-71.

15. Amsterdam EA, Wenger NK, Brindis RG, Casey DE Jr, Ganiats TG, Holmes DR Jr, et al; ACC/AHA Task Force Members. 2014 AHA/ACC guideline for the management of patients with non-ST-elevation acute coronary syndromes: a report of the American College of Cardiology/American Heart Association Task Force on Practice Guidelines. Circulation. 2014;130:e344-426.

16. Kafkas N, Venetsanou K, Patsilinakos S, Voudris V, Antonatos D, Kelesidis K, et al. Procalcitonin in acute myocardial infarction. Acute Card Care. 2008;10:306.

17. Erren M, Reinecke H, Junker R, Fobker M, Schulte H, Schurek JO, et al. Systemic inflammatory parameters in patients with atherosclerosis of the coronary and peripheral arteries. Arterioscler Thromb Vasc Biol. 1999;19:2355-63.

18. Waldo SW, Brenner DA, Li S, Alexander K, Ganz P. Reperfusion times and in-hospital outcomes among patients with an isolated posterior myocardial infarction: Insights from the National Cardiovascular Data Registry (NCDR). Am Heart J. 2014;167:350-4.

19. Picariello C, Lazzeri C, Chiostri M, Gensini G, Valente S. Procalcitonin in patients with acute coronary syndromes and cardiogenic shock submitted to percutaneous coronary intervention. Intern Emerg Med. 2009;4:403-8.

20. Günal Ö, Ulutan F, Erkorkmaz Ü. Prognosticity of procalcitonin in patients with sepsis. Klimik Journal. 2011;24:31-5.
21. Senturk T, Cordan J, Baran I, Ozdemir B, Güllülü S, Aydinlar A, et al. Procalcitonin in patients with acute coronary syndrome: correlation with high-sensitive Creactive protein, prognosis and severity of coronary artery disease. Acta Cardiol. 2007;62:135-41.

22. Ridker PM. Clinical application of C-reactive protein for cardiovascular disease detection and prevention. Circulation. 2003;107:363-9.

23. Kelly D, Khan SQ, Dhillon O, Quinn P, Struck J, Squire IB, et al. Procalcitonin as a prognostic marker in patients with acute myocardial infarction. Biomarkers. 2010;15:325-31.

24. Sinning CR, Sinning JM, Schulz A, Schnabel RB, Lubos E, Wild PS, et al; AtheroGene Study Investigators. Association of serum procalcitonin with cardiovascular prognosis in coronary artery disease. Circ J. 2011;75:1184-91.

25. Antman EM, Tansijevic MJ, Thompson B, Schactman M, McCabe CH, Cannon CP, et al. Cardiac-spesific troponin llevels to predict the risk of mortality in patients with acute coronary syndromes. New Engl J Med. 1996;335:1342-9.

26. Rao ACR, Collinson PO, Canepa-Anson P, Josepb SP. Troponin T measurement after myocardial infaretion can identify left ventricular ejection of less than 40\%. Heart. 1998;80:223-5.

27. Remskar M, Horvat M, Hojjer S, Noc M. Procalcitonin in patients with acute myocardial infarction. Wien Klin Wochenschr 2002;114:205-10.

28. Cupples LA, Gagnon DR, Wong ND, Ostfeld AM, Kannel WB. Preexisting cardiovascular conditions and long-term prognosis after initial myocardial infarction: the Framingham Study. Am Heart J. 1993;125:863-72. 